\title{
Probing the inner jet of M87; from the jet base to HST-1
}

\author{
Kazuhiro Hada ${ }^{1,2, a}$ \\ ${ }^{1}$ INAF Istituto di Radioastronomia, Via Gobetti 101, 40129 Bologna, Italy \\ ${ }^{2}$ National Astronomical Observatory of Japan, Osawa, Mitaka, Tokyo 181-8588, Japan
}

\begin{abstract}
The giant radio galaxy M87 accompanies one of the nearest Active-Galactic-Nuclei jets showing the intense radiation through radio to $\mathrm{TeV}$ gamma-ray. Its proximity and the large mass of the central black hole provide an excellent advantage to probe the sites of jet formation and gamma-ray production. Here we review some of our recent studies for the inner jet of M87 based on the multi-frequency and multi-epoch VLBI observations, especially focusing on the two remarkable regions i.e., the jet base near the black hole and the peculiar feature HST-1. Our multi-frequency observations with the phase-referencing technique revealed the detailed structure of the jet base region regarding the location of the central engine and the collimation profile. In terms of HST-1, the intense multi-epoch VLBI monitoring constrained the accurate kinematic properties and the structural variations in this complicated feature, together with a possible connection to the gamma-ray activities. At the end of this contribution, we briefly describe our new monitoring project for M87 with VERA, which permits a detailed study on the structural evolution in the jet base region.
\end{abstract}

\section{Introduction}

Powered by the accretion of material onto supermassive black holes, relativistic jets in Active Galactic Nuclei (AGN) are the most energetic phenomena in the Universe. Understanding of their formation, collimation, acceleration as well as the production processes of high-energy emission up to $\mathrm{MeV}, \mathrm{GeV}$ and $\mathrm{TeV}$ is one of the major goals in current astrophysics.

Over the past years, remarkable progress has been made in the theoretical field of jet formation, and some of numerical simulations are beginning to resolve the detailed processes of jet launching from the black hole/accretion disk system as well as the large-scale propagation [16]. Many of current studies favor the framework of the magnetically-driven jet, where the evolution of the jet dynamics is subject to the efficient conversion of magnetic energy into kinetic energy. The acceleration of the bulk flow is effected by magnetic pressure gradients or magneto-centrifugal forces, while the collimation is achieved by magnetic hoop stresses and/or the pressure support from the external medium at the jet boundary, resulting in a high Lorentz factor jet. Eventually, at a specific location in the propagating jet, some of the gained kinetic energy is dissipated through shocks or other dissipative processes, creating a potential site of high-energy gammaray emission $[7,8]$. In order to test these models, direct comparisons with observations at a matched scale are essential. However, those active processes are expected to take place within the very inner part of jet i.e., typically

\footnotetext{
ae-mail: hada@ira.inaf.it
}

within $10^{2} \sim 10^{5}$ Schwarzschild radii $\left(R_{\mathrm{S}}\right)$, leaving only a few potential sources to realize the direct comparisons.

At a distance of 16.7 Mpc [9], the nearby radio galaxy M87 (Virgo A, 3C 274, NGC 4486) is associated with one of the best studied relativistic jets. Hubble Space Telescope (HST) spectroscopy of its nuclues has found a rapidly-rotating ionized gas disk at its center [10-12], providing strong evidence for the presence of a central supermassive black hole weighing $M_{\mathrm{BH}} \sim(2-4) \times 10^{9} M_{\odot}$. Recent analyses of the steller kinematics suggest its mass about twice larger i.e., $M_{\mathrm{BH}} \sim 6 \times 10^{9} M_{\odot}[13,14]$, although this remains a matter of debate [15]. The jet on arcsecond scale is characterized by several remarkable knots, the innermost ( $\sim 1$ arcsec from the nucleus) feature of which is known as HST-1 [17]. The sufficient brightness of the jet has enabled detailed studies at radio, optical and X-ray over years [e.g., 16-18]. With the advent of Fermi and a set of high sensitivity Cherenkov telescopes, M87 is now rotinely detected up to $\mathrm{MeV}, \mathrm{GeV}$ and $\mathrm{TeV}$ gammaray $[19,20]$. Owing to the combination of its proximity and large inffered black hole mass, 1 milliarcsecond (mas) attains $0.08 \mathrm{pc}$ or $140 R_{\mathrm{s}}$ (for $D=16.7 \mathrm{Mpc}$ and $\left.M_{\mathrm{BH}}=6 \times 10^{9} M_{\odot}\right)^{1}$. M87 thus provides a unique opportunity to study the relativistic-jet phenomena on an unprecedented scale.

The detailed structure of the inner region of the M87 jet has been intensively investigated with Very-LongBaseline-Interferometry (VLBI) observations (Figure 1). A pioneering work by Junor et al. [25] at $43 \mathrm{GHz}$ discovered a strongly edge-brightened structure with a broaden-

\footnotetext{
${ }^{1}$ In this contribution we adopt these values for the distance and the black hole mass of M87.
} 


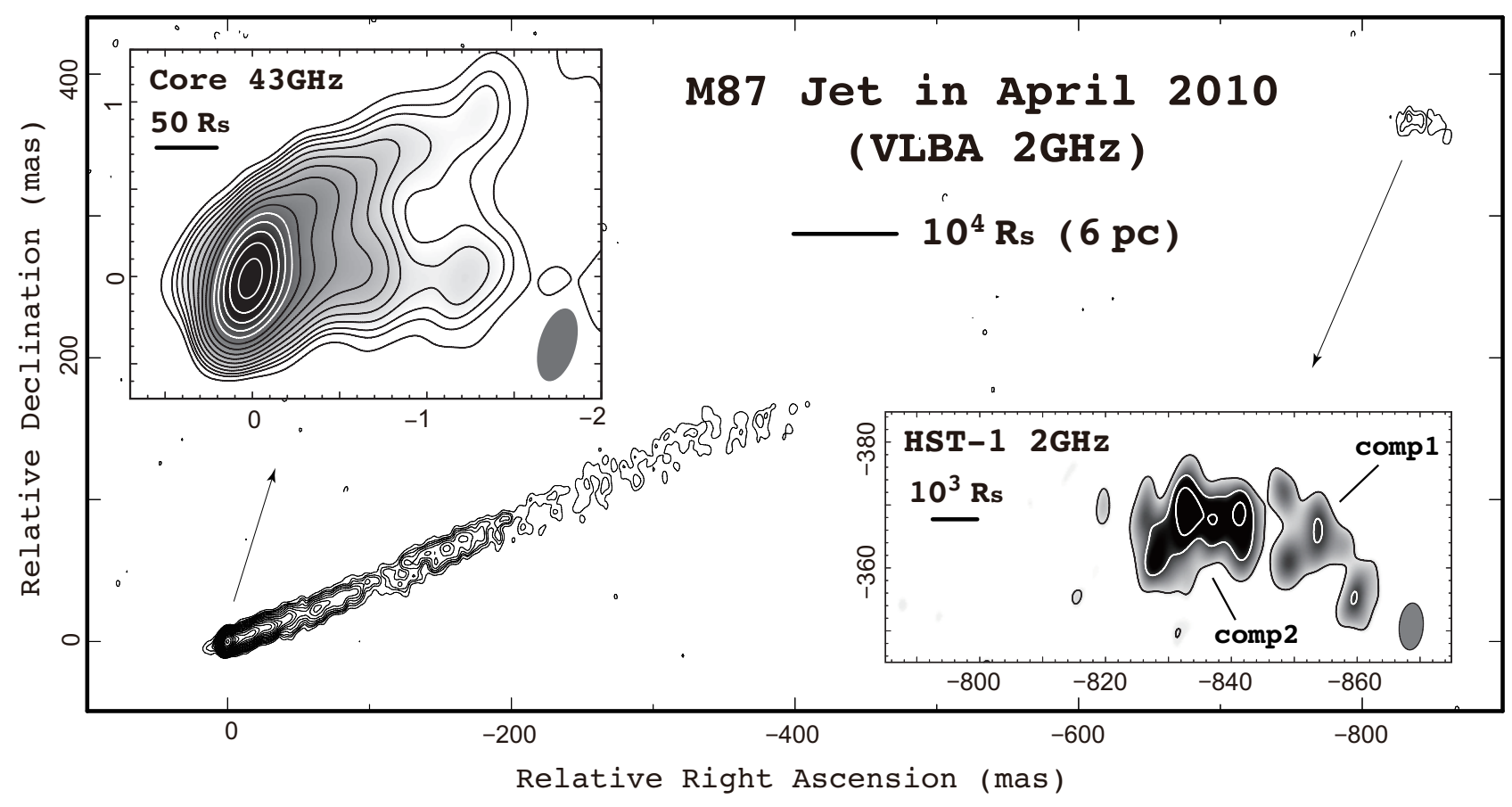

Figure 1. Summary of VLBA images of the M 87 jet (taken from [42]). The main (global) image is obtained at 2 GHz. The bottom right inset indicates a close up view toward the HST-1 region. The nomenclatures of two main features (comp1 and comp2) are based on [43]. The upper left inset indicates a $43-\mathrm{GHz}$ image for the core and the inner jet. The beam sizes at $2 / 43 \mathrm{GHz}$ are $7.5 \times 3.9 \mathrm{mas}$ in P.A. $-5^{\circ}$ (bottom right in the inset of HST-1) and $0.43 \times 0.21$ mas in P.A. $-16^{\circ}$ (bottom right in the $43-\mathrm{GHz}$ image), respectively. For each image, contours start from $-1,1,2 \ldots$ times $3 \sigma$ image rms levels $\left(3 \sigma=1.0 / 3.3\right.$ mJy beam $^{-1}$ at $\left.2 / 43 \mathrm{GHz}\right)$ and increasing by factors of 1.4 .

ing of the opening angle near the radio core. Such a structure was later confirmed by several authors [26-30]. Recently, Asada \& Nakamura [31] found a maintenance of a parabolic jet shape between $\sim 100$ and $10^{5} R_{\mathrm{s}}$ from the jet base with a transition into a conical shape above $10^{6} R_{\mathrm{s}}$. On the smallest scale, the recent VLBI experiment at $230 \mathrm{GHz}$ revealed a compact structure that is comparable to the size of the event horizon or the inner part of accretion disk [32]. These provide evidence that the jet launch and collimation regions are beginning to be resolved in this jet.

The kinematic properties and the structural evolution of this jet remain rather elusive. Near the jet base, a variety of jet speeds have been claimed ranging from quasistationary $(<0.05 c)$ [30], to subluminal $(0.25-0.4) c[28$, 33 ] and to superluminal $(\sim 2 c)$ speeds [21]. On larger scale, the optical observations measured a highly superluminal ( $\sim 6 c)$ speed in HST-1 [17]. Superluminal components at a slightly slower speed $(\sim 4 c)$ were measured in HST-1 by VLBI observations [34]. However, another VLBI monitoring of this feature argue a subluminal motion [35]. Moreover, the exsistence of a stationary feature is also suggested at the upstream side of HST-1 [34], which is claimed as a recollimation shocked feature [34, 36].

In terms of the high-energy activity, M87 often shows strong flaring events at $\mathrm{TeV}$ with time-scales of the order of a few days [20]. The location and the origin of these $\mathrm{TeV}$ events are one of the major questions for the M87 jet. In the active episode occurred in 2005 [23], the $\mathrm{TeV}$ flare was accompanied by radio-to-X-ray flares from
HST-1 together with the emergence of superluminal radio components [34]. These lead to the strong argument that the HST-1 region is associated with the gamma-ray production site [34, 36-38]. On the other hand, such a rapid variability implies an extremely compact emitting volume of less than $\sim 5 \times 10^{15} \delta \mathrm{cm}$ or $5 \delta R_{\mathrm{s}}$ [23], suggesting the nuclues of M87 itself as a likely site of TeV emission. In fact, the second $\mathrm{TeV}$ event occurred in 2008 accompanied with a strong flux increase of the radio core and also the X-ray core, while HST-1 maintained a comparatively constant flux [21]. A remarkable TeV flare also occurred in 2010 [20], where the X-ray observations detected an enhanced flux from the core whereas HST-1 remains a low state [39].

As overviewed through the above paragraphs, our observational understanding for the M87 jet has indeed progressed significantly during the past decade. However, it is also true that some of the fundamental aspects, including the jet kinematics and the gamma-ray properties as well as the jet base structure, still remain poorly constrained. To better understand the M87 jet, we have recently been performing several VLBI projects that are dedicated for M87. In [40], we investigated the location of the central engine of the M87 jet using multi-frequency, accurate astrometric observations. Following this study, in [41] we revealed the detailed collimation profile of the M87 jet over the wide range of distance down to a scale of $10 R_{\mathrm{s}}$. We also probed the detailed radio status of the jet base during the $2010 \mathrm{TeV}$ flare in [42]. On the other hand, in 


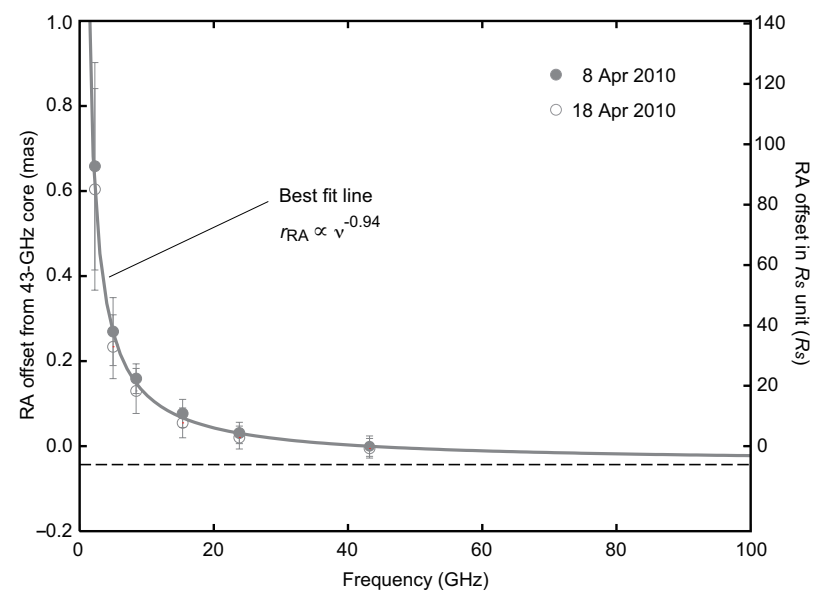

Figure 2. Plot of the core-shift measurements in right ascension for M 87 as a function of observing frequency (taken from [40]). The data sets of filled and open circles are results for 8 and 18 April, respectively. Both observations were made at 2.3, 5.0, 8.4, $15.4,23.8$ and $43.2 \mathrm{GHz}$. The origin of the vertical axis is referenced to the weighted-mean position of the $43.2-\mathrm{GHz}$ core over the two epochs. This plot shows that the measured core positions for the two epochs are consistent within 1s error bars, indicating that the systematic errors are effectively cancelled out through the quasi-simultaneous multi-frequency observations. The solid curve represents the best-fit solution, with $r_{\mathrm{RA}}(v) \propto A v^{-a}+B$ ( $a=-0.94 \pm 0.09, A=1.40 \pm 0.16$ and $B=-0.041 \pm 0.012$ ), which is derived from the weighted least-square method to the entire data set. The dashed horizontal line represents the asymptotic line of the solid curve, which is located at $41 \mu$ as eastwards from the $43.2-\mathrm{GHz}$ core in RA.

[43] we constrained accurately the structural evolution of HST-1 with the dense monitoring with the European VLBI Network (EVN). These results would improve our understanding for this jet.

In this contribution, we overview these results. We firstly describe the jet base structure that was revealed based on the multi-frequency observations. We then describe a deep monitoring project of HST-1 with EVN. At the end of this contribution, we also briefly present our new monitoring project with the VLBI Exploration of Radio Astrometry (VERA), which permits a detailed study toward the jet base region.

\section{The structure of the innermost jet}

\subsection{Location of the M87 core}

In Junor et al. [25], their argument of the jet launch with the wide opening angle was based on the implicit assumption that the location of the central engine of M87 is coincident with the core. However, this issue is not so simple. Since one can only observe the jet base up to the surface where the optical depth for synchrotron-selfabsorption $\left(\tau_{\mathrm{ssa}}\right)$ becomes unity [44], radio cores generally can be offset from the central engine. A stationary standing shock also can be an apparent radio core if it is more intensive than the $\tau_{\text {ssa }} \sim 1$ surface [45]. Recent studies of several blazars indicate that the radio cores in these

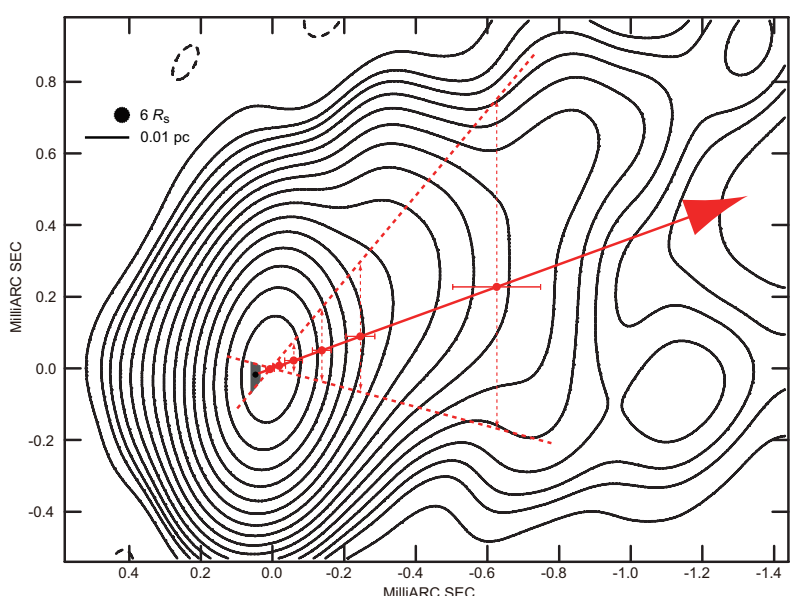

Figure 3. VLBA image of M 87 at $43 \mathrm{GHz}$ superimposed on the measured core shift positions (taken from [40]). Contours are $(-1,1,2,2.8$ and 4$) \times 3.3 \mathrm{mJy}$ per beam and thereafter increase by factors of $2^{1 / 2}$. The synthesized beam of the VLBA is 0.22 mas $\times 0.46$ mas at $-5^{\circ}$. The peak brightness and $1 \sigma$ noise level are $724 \mathrm{mJy}$ abd $1.1 \mathrm{mJy}$ per beam, respectively. Two broken red lines represent the maximum possible range of the inner jet direction centred on the $43.2-\mathrm{GHz}$ core. A solid red arrow represents the larger-scale jet direction. Red circles indicate the core positions at $2.3,5.0,8.4,15.2,23.8$ and $43.2 \mathrm{GHz}$ relative to the $43.2-\mathrm{GHz}$ core (the higher the frequency of the core, the closer it approaches the central engine). The shaded area at the east of the 43.2-GHz core represents the upstream end of the jet derived from the coreshiftmeasurements. This area is enclosed by the $1 \sigma$ error in the core-shift value in RA, and the direction of the inner jet defines uncertainties in declination.

jets are located about $10^{4} \sim 10^{6} R_{\mathrm{s}}$ downstream of the central engines [7, 46-48]. Constraining the relative distance between the core and the central engine is crucial for correctly measure the opening angle and the collimation profile at the jet base [28]. In the case of M87, several authors argue detections of the "counter feature" at the eastern side of the radio core with a possbile outward motion $[27,28,30]$. If this is the case, the counter feature is actually the counter jet and the central engine should be located in the immediate vicinity of the core.

Alternatively, this issue can be tested by multifrequency VLBI observations with the phase-referencing technique. If the radio core corresponds to an opticallythick surface of SSA, the core should move toward the central engine at higher frequencies because the jet becomes more transparent $[49,50]$, and eventually approach the upstream end of the jet, which is supposed to be the immediate vicinity of the central engine. The core shift effect has recently been observed for a growing number of sources [51-54]. Our strategy is to measure such a frequency dependence of the core shift by using the accurate phase-referencing technique, which enables us to perform astrometric measurements with positional accuracies of down to tens of micro-arcseconds ( $\mu \mathrm{as})$ relative to a stationary position reference. This angular accuracy corresponds to a gravitational scale below $10 R_{\mathrm{S}}$ for M87, 


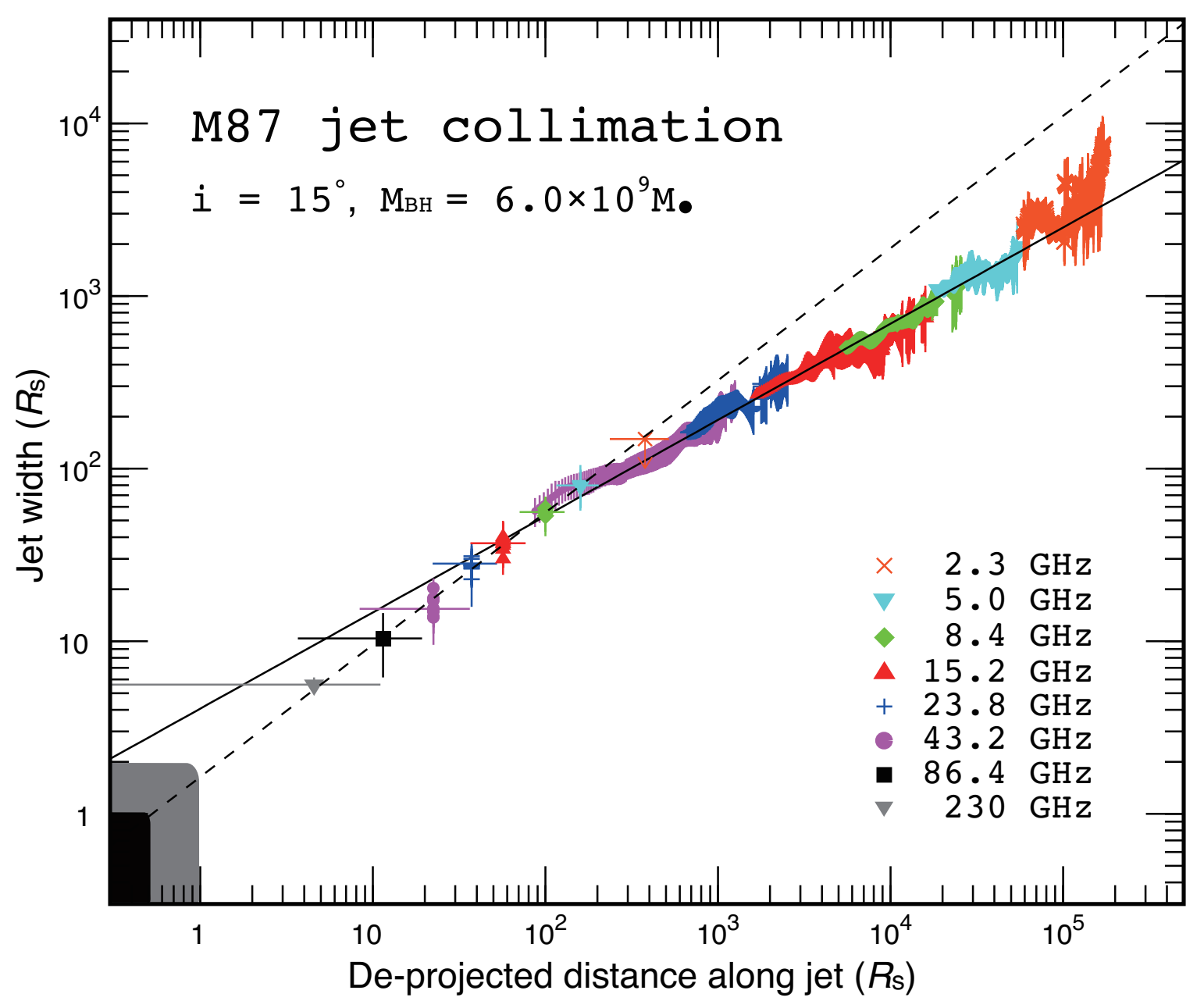

Figure 4. Jet width profile of M87 as a function of distance (taken from [41]). A jet viewing angle of $i=15^{\circ}$ and a black hole mass of $M_{\mathrm{BH}}=6.0 \times 10^{9} M_{\odot}$ are adopted. Densely-sampled part indicates the width profile of the edge-brightened jet measured at multiple frequency with errors. Discretely-sampled part indicates the FWHM size profile for the core region projected in P.A $=20^{\circ}\left(W_{\mathrm{c}}\right)$. The core positions and the errors are estimated based on the previous astrometry result [40]. A solid line indicates the best fit solution for the edge-brightened jet width $W_{\mathrm{j}} \propto r^{0.56}$, while a dashed line indicates $r^{0.76}$. The grey/black rectangles at the bottom left corner of the figure represent surfaces (event horizons) for non-spinning/maximally-spinning black holes, respectively.

which is comparable to the diameter of the innermost stable circular orbit for a non-rotating black hole $\left(6 R_{\mathrm{s}}\right)$.

In Figure 2, we show the result of our multi-frequency astrometry toward the M87 core [40]. We clearly detected core shifts between 2 and $43 \mathrm{GHz}$, indicating that the M87 core is actually the optically-thick surface among these frequencies. The most remarkable finding is that the core shift asymptotically converges to a point only $41 \mu$ as upstream of $43-\mathrm{GHz}$ core, corresponding to a projected separation of $6 R_{\mathrm{S}}$ on the sky. This result is actually consistent with the previous detections of the counter jet and its eastward motion [28, 30], and eventually confirmed the argument of the wide opening angle jet production claimed by [25] (Figure 3).

The resultant separation between the radio core and central engine for M87 is much smaller than those independently estimated for blazars BL Lac, PKS 1510-089, OJ 287, and 3C 279 on the basis of the combinied observations such as the multi-band (radio to $\gamma$-ray) lightcurves, VLBI monitorings and the rotations of the optical po- larization angle [7, 46-48]. As a possible explanation, this discrepancy could be related to the structured jet scenario [55], where the core emission of blazars comes from the faster spine part brightening at a large distance, while the core of the misaligned jet M87 originates in the slower sheath part near the black hole. It is interesting to note that the violent feature HST-1 is located at a de-projected distance of $\gtrsim 2 \times 10^{5} R_{\mathrm{s}}$, the detailed properties of which are described in the next section. Since this feature exhibits the remarkable multi-wavelength flaring event that accompanies the superluminal ejections, the HST-1 region could be associated with the activities of blazar cores.

We note that, as stated in [40], our argument here is based on the assumption that the central engine is located at the convergence point of the core shift. We do not rule out the possibility that there is a slight offset between the black hole position and the convergence point of the core shift. However, our recent measurements of core shifts with the phase-referencing technique toward NGC 4261, which is a similar FR-I type source to M87 but has a well- 
defined two-sided jets, reveal that the core shifts for both the jet and the counter-jet converges toward the same point on the sky, which is consistent that the central engine is actually coincident with the convergence point of the core shift (Haga et al. in preparation).

\subsection{Jet collimation profile of the innermost region}

The work in presented in [40] has important implications in the following respects. First, the constraint on the central engine location allows us to probe the radial profile of the jet structure as a function of the distance from the central engine. Second, the determination of the frequency dependence of the core position $r_{\mathrm{c}} \propto v^{-0.94 \pm 0.09}$ enables us to reveal the structure of the innermost jet by investigating the multi-frequency properties of the core. Hence, by combining the radial properties of the core and the resolved jet, we can reveal the detailed jet structure over the wide range of distance down to the immediate vicinity of the black hole.

In Figure 4, we show the radial profile of the M87 jet width as a function of (de-projected) distance along jet (assuming a jet viewing angle $i=15^{\circ} ;[17,56]$ ), which was made by analyzing many VLBA data between 2 and $86 \mathrm{GHz}$ [41]. Here we show two kinds of radial profiles; the first one $W_{\mathrm{j}}$ is a radial distribution for the edgebrightned jet (outer part) and the other one $W_{\mathrm{c}}$ represents a radial distribution of the core size transverse to the jet (inner part). We confirmed that the edge-brightened region is well-expressed as a parabolic structure of $W_{\mathrm{j}}(r) \propto r^{0.56 \pm 0.03}$ (solid line in Figure 4). This is in good agreement with the independent measurement of $r^{0.58 \pm 0.02}$ by [31]. For the region around $\sim 100 R_{\mathrm{s}}$, where the independent measurements of $W_{\mathrm{j}}$ and $W_{\mathrm{c}}$ overlap with each other, $W_{\mathrm{c}}$ at 5 and $8,4 \mathrm{GHz}$ smoothly connect with $W_{\mathrm{j}}$ at $43 \mathrm{GHz}$, indicating that $W_{\mathrm{c}}$ is actually a good tracer for the width of the innermost jet. The combination of the core size $\left(W_{\mathrm{c}} \propto v^{\xi}\right.$ where $\xi=-0.71 \pm 0.05 ;[41])$ and core position $\left(r \propto v^{\alpha}\right.$ where $\alpha=-0.94 \pm 0.09$; [40]) yields a radial dependence of $W_{\mathrm{c}}$ as $W_{\mathrm{c}}(r) \propto r^{\frac{\xi}{\alpha}}=r^{0.76 \pm 0.13}$ (dotted line in Figure 4), which is slightly steeper than that of the outer jet $W_{\mathrm{j}}(r)$, although the uncertainty is still large. In the present result, it is still difficult to distinguish whether $W_{\mathrm{c}}$ at $5,8.4$, 15 and $23.8 \mathrm{GHz}$ are on the solid line or the dashed line due to their position uncertainties as well as those of sizes. On the other hand, $W_{\mathrm{c}}$ at 43 and $86 \mathrm{GHz}$ tends to be below the solid line. In Figure 4 we also plot the core size at $230 \mathrm{GHz}$ that was measured by the recent mm-VLBI experiment [32]. However, the exact profile cannot be discriminated again because the data is totally dominated by its position uncertainty at this frequency.

This is the first work reavealing the detailed collimation profile of the M87 jet down to $\sim 10 R_{\mathrm{s}}$ scale. An intriguing point here is that the measured radial profile near the black hole suggests a possible change in the jet collimation profile from the outer parabolic one, where the jet shape tends to become more radially oriented. A transition of jet collimation profile near the black hole is actually suggested from some of theoretical aspects. In the framework of relativistic MHD jet models, most of the energy conversion from magnetic-to-kinetic occurs after the flow passes through the fast-magnetosonic point (the "magnetic nozzle" effect; [57, 58]). Beyond this point, the magnetized jet starts to be collimated asymptotically into a parabolic shape, because the increasing plasma inertia winds the field lines into toroidal directions and thus amplifies the hoop stresses [e.g., 59]. The radius of the fast point is typically a few times the light cylinder radius $R_{\mathrm{lc}}$ [57], where $R_{\mathrm{lc}}$ is of the order of (1 5) $R_{\mathrm{S}}$ [5]. Thus, if the M87 jet is magnetically launched, the observed possible transition of the jet shape around $10 \sim 100 R_{\mathrm{s}}$ could be explained by this process. Moreover, the jet in this scale is likely to have complicated interactions with surrounding medium, including accretion flows, coronae and disk winds [e.g., 4]. Their geometries and the local pressure balance at the jet boundary would affect the initial jet shape. Alternatively, such a change of the jet shape could occur as an apparent effect due to projection, if the jet viewing angle is not constant down to the black hole [60].

The result presented here should be examined more rigorously in future higher-resolution/higher-senstivity observations. To this end, we are going to perform new high-sensitivity VLBA observations at $86 \mathrm{GHz}$ in combination with quasi-simultaneous sessions at lower frequencies, which allows more robust investigations of the jet structure within $100 R_{\mathrm{s}}$ and thus tests some specific models more quantitatively. Moreover, the accurate determination of the jet collimation profile in this scale will be able to yeild the constraint on the size of the jet footprint by extrapolating the profile toward the entral engile, which allows us to estimate the spin parameter of the jet-launching black hole [32].

\section{EVN monitoring of HST-1}

Located at a de-projected distance of at least $120 \mathrm{pc}$ or $2 \times 10^{5} R_{\mathrm{s}}$ from the jet base, the peculiar feature HST- 1 may provide a clue for understanding the processes of jet formation and gamma-ray production of M87. The multiwavelength flaring event in 2005, where the superluminal ejections occurred coincidentally, are reminiscent of the blazar activity [34]. However, the detailed structure and the kinematic properties of the sub-structures in HST-1 remains highly controvertial [35], requring more dedicated VLBI studies toward this feature.

From mid 2009, we started a deep monitoring program of HST-1 with EVN at $5 \mathrm{GHz}$. The aim of this project is to constrain the detailed structural evolution of the HST1 region as well as the inner jet, and moreover to clarify the physical connection to activities at $\mathrm{TeV}$ gamma-ray. In fact, M87 underwent a large TeV flare in 2010 [20] and our EVN project successfully covered this episode. The chosen array configuration provides a suitable combination of resolution, sensitivity, and field of view, which permits a detailed study of the behaviour of the HST-1 region.

Figure 5 surmmarizes the first outcome of this project [43]. In addition to the EVN data, we also extend back to the past by including many VLBA archival data 


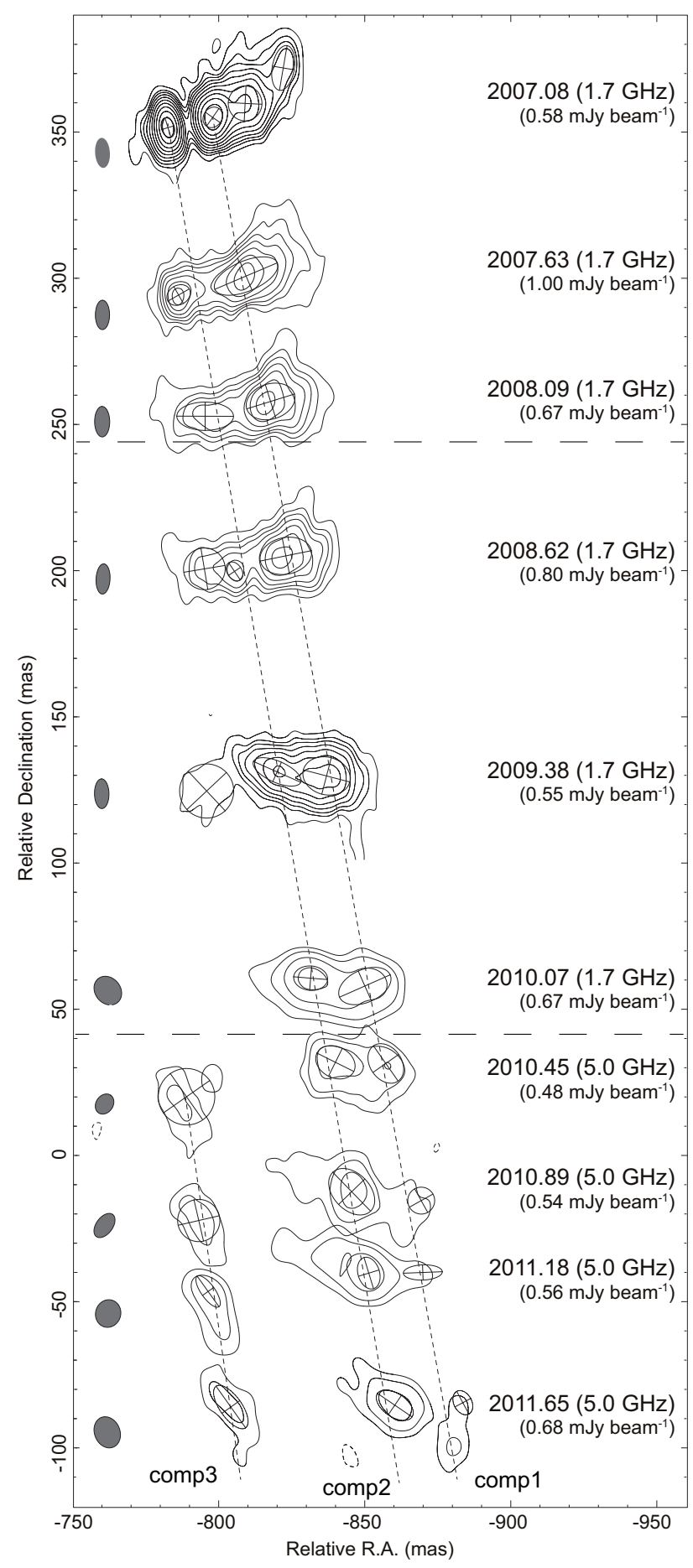

Figure 5. Set of HST-1 images (taken from [43]). For each epoch, we give the epoch, frequency, lowest contour to the right, and the restoring beam to the left of the respective contour plot. Contours are traced at $(-1,1,2,4 \ldots)$ multiples of the given value. The model-fit components are overlaid as ellipses with crosses. The contour plots are spaced vertically proportionally to the time interval between the relative epochs. Two horizontal long-dashed lines indicate the epochs of the recent VHE $\gamma$-ray flares in 2008 and 2010. The axes represent the relative (RA, Dec) coordinates from the core for the first image.

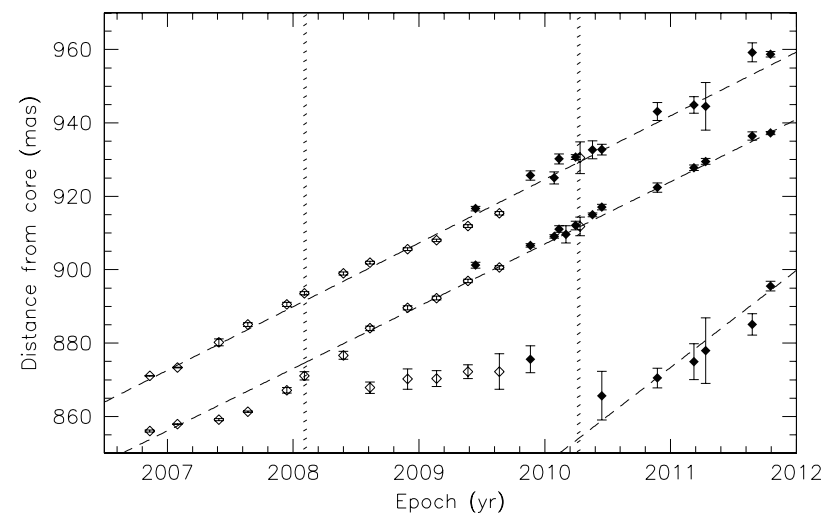

Figure 6. Distance of the HST-1 sub-components from the core versus time (taken from [43]). Empty and filled symbols show 1.7 (VLBA) and $5 \mathrm{GHz}$ (EVN) data, respectively. The dashed lines show least-squares linear fits, while the vertical stripes represent the epochs of the 2008 and 2010 VHE flares.

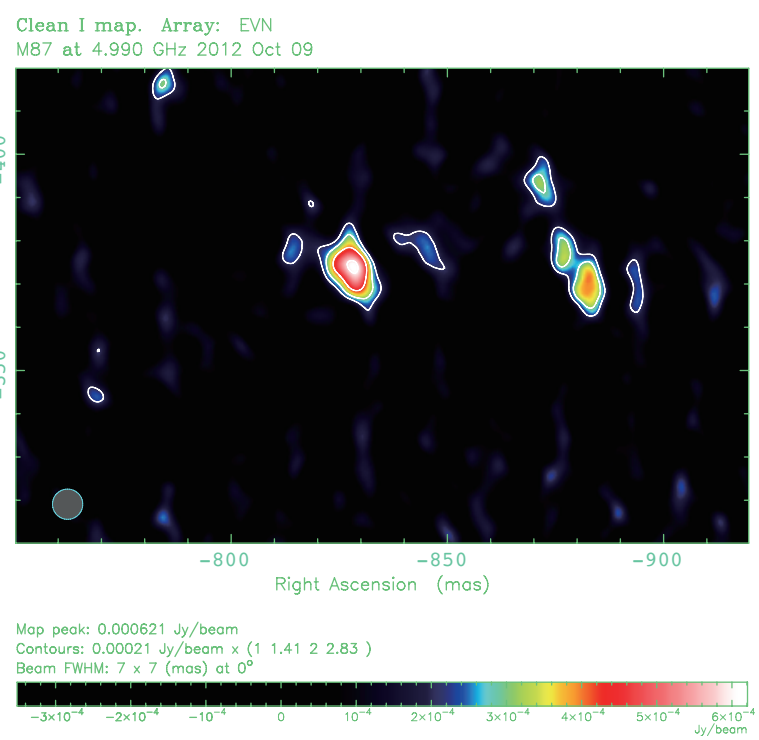

Figure 7. The latest e-EVN image of HST-1 at $5 \mathrm{GHz}$ (October 2012). The image is convolved with a 7-mas-diameter circular beam. Contours starts from 3 times the image rms level. The axes are the relative coordinates from the core. The brightest region at the upstream side corresponds to the comp3, while the visible downstream region traces the comp2.

1.7 $\mathrm{GHz}$, which allows us to track the detailed evolution of HST-1 over more than 5 years. We detect significant flux density in the HST-1 region at all epochs, in addition to the bright core and the inner jet. The HST-1 region extends for over 50 mas and is resolved in complex substructures. The overall position angle and the location of the individual substructures evolve with time. In terms of the kinematic properties of HST-1, we found the following remarkable facts during this period:

\section{- Significant proper motions over 5 years}

Thanks to the many observations and the good accuracy in fitting the structure, we can reliably track the components between the various epochs (see Figure 6). 
In particular, the identification of the two main components are significant, which we label as component 1 and 2 , of which 1 is the outermost. These have moved by very similar distances over 5 years between 2006.86 and $2011.80\left(\Delta r_{1}=88.1 \mathrm{mas}, \Delta r_{2}=81.2 \mathrm{mas}\right)$, corresponding to apparently superluminal velocities around $\beta_{\text {app }}=4.1$. The uncertainty on this superluminal value can be constrained down to as small as a few percent thanks to the number of observations.

\section{- Change of P.A.}

At the early epochs, an overall position angle of the HST-1 region is oriented similarly to the main jet of M $87\left(\sim-65^{\circ}\right)$. In the following epochs, the position angle progressively rotates to $\sim-90^{\circ}$ and finally to $\sim-100^{\circ}$ as the components move outward (see also Figure 3 in [43]).

\section{- Structural variation and a new component emer- gence}

The size of each component varies and additional components are present at some epochs. In particular, component 2 becomes quite extended in early 2008 and eventually splits into two components from 2008.62. After this split, the upstream subcomponent remains more or less stationary and gradually becomes fainter. When the $5 \mathrm{GHz}$ observations start, there is little evidence of this component. However, starting from 2010.45, a new inner component is again required to fit the $5 \mathrm{GHz}$ data. This component appears consistently thereafter and we name it component 3 . This substructure is also moving superluminally, although the uncertainty is larger because of the shorter time range. The epochs of these structural variations are similar to those of the $\mathrm{TeV}$ events reported in 2008 and 2010.

These results are quite robust and improve our observational understanding for the nature of HST-1. From the observed superluminal speed $\sim 4 c$ of the two main components, we can infer a range of the possible intrinsic jet velocity, assuming that the pattern and bulk velocity are the same. Adopting a jet orientation angle in the range $15^{\circ}<\theta<25^{\circ}$ (e.g. [21]), the measured apparent velocity corresponds to an intrinsic velocity $0.97 c<v<0.99 c$, which in turn implies a Doppler factor and a Lorentz factor for this structure between $\delta_{\mathrm{HST}-1}=1.5$ and $\Gamma_{\mathrm{HST}-1}=6.5$ (for $\theta=25^{\circ}$ ), and $\delta_{\mathrm{HST}-1}=3.9$ and $\Gamma_{\mathrm{HST}-1}=4.1$ (for $\theta=15^{\circ}$ ), respectively. This result agrees with the synchrotron model for the X-ray emission discussed by Marshall et al. [63] and Harris et al. [64].

At first sight, our result does not support the identification of HST-1 as a standing-shock structure as originally proposed by $[34,36]$, given the displacements of $>80$ mas found for components 1 and 2 over $\sim 5$ years. Moreover, no prominent stationary components were consistently detected in the 26 observations considered in this work. However, the components change in size over time, and in particular component 2 splits into substructures after 2008.62, suggesting that other features exist in HST-1 and interact with the brightest knots. Those components may be underlying, standing or very slowly moving re-

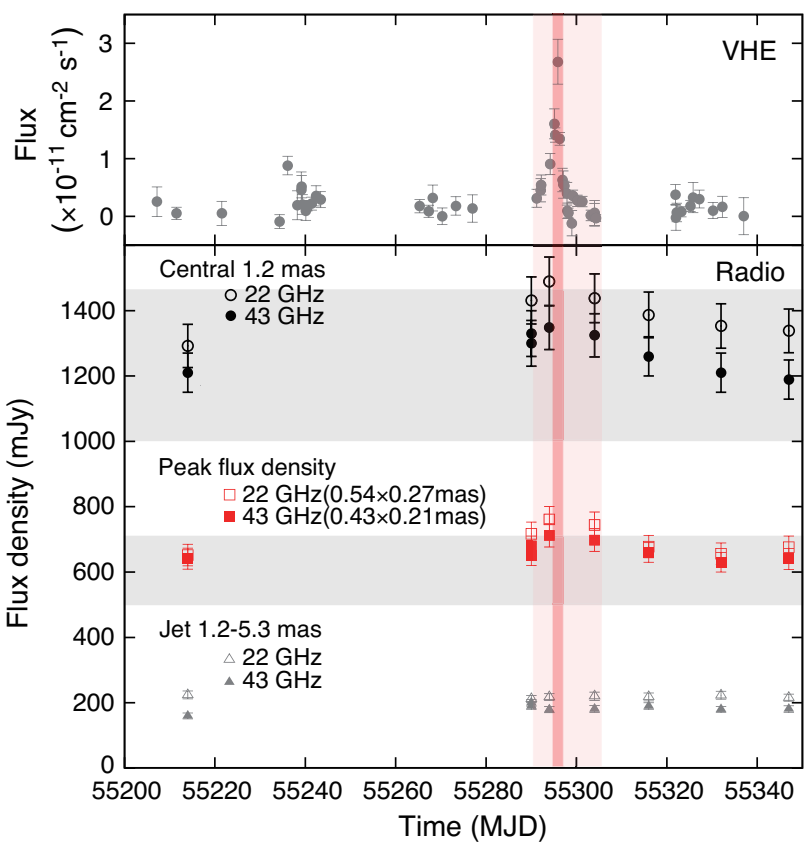

Figure 8. 43- and 22-GHz lightcurves of the central region of M 87 near the VHE flare in April 2010 (taken from [42]). The fluxes for three different regions (i) the central 1.2 mas, (ii) the peak, and (iii) the outer jet are provided. The light red area indicates the period where the $\mathrm{TeV} \gamma$-ray flaring event was covered by the $\mathrm{TeV}$ telescopes. The dark red area indicates the date of the $\mathrm{TeV} \gamma$-ray flux peak (MJD 55296). For reference, we show the ranges of the flux densities at $43 \mathrm{GHz}$ before the 2008 flare for the regions (i) and (ii), which represent typical flux ranges for the quiescent phase [21]

gions, too faint to be detected separately but contributing to the total emission when brighter components are nearby. In particular, this could be the case for the upstream-edge component "D" denoted by Cheung et al. [34], which only becomes visible when a new feature (like our component 3 ) is ejected/created within HST-1. Moreover, this region seems to be related to a transition of the parabolicto-conical jet shape with a overpressured signature [31]. Such a behavior of the D component would support the scenario in which HST-1 is indeed a stationary reconfinement shock structure.

The EVN monitoring program is still actively going on (Figure 7). Since the recent images of HST-1 at $5 \mathrm{GHz}$ indicates a constant decrease of the overall brightness, we switch the observing frequency to $1.7 \mathrm{GHz}$ from the season of 2013, which permits higher sensitivity detections thanks to a steep spectral nature of HST-1 [43]. These data will allow deeper investigations for the possible recollimation shocked region, the kinematics of the superluminal features and also additional discussions in terms of the lightcurves and the trajectories for the individual subcomponents.

\section{The jet base monitoring with VERA}

The coincidence of the observed structural variations and the $\mathrm{TeV}$ events during our HST-1 monitoring is particu- 


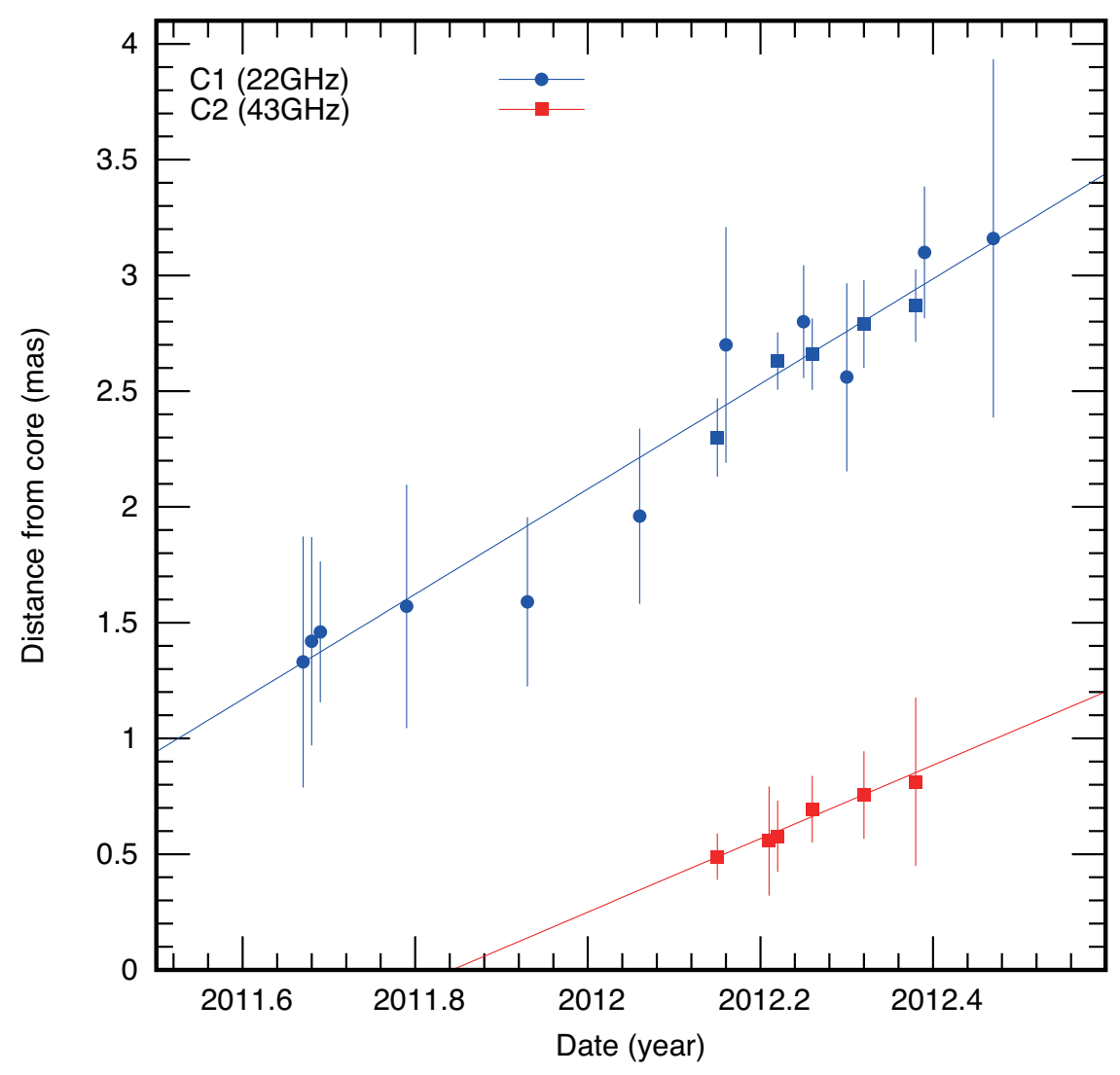

Figure 9. Preliminary result on proper motion measurements near the jet base with the VERA monitoring project at 22 and $43 \mathrm{GHz}$ (Hada et al. in prep). The horizontal axis indicates time, while the vertical axis represents radial distance from the radio core. A set of the blue data indicates the evolution of a knot detected at $22 \mathrm{GHz}$ (here we call $\mathrm{C} 1$ ), while a set of the red points represents the motion for another feature detected at $43 \mathrm{GHz}$ (we denote $\mathrm{C} 2$ ). Our preliminary measurements derive sub-luminal motions of $0.6 c$ for $\mathrm{C} 1$ and $0.4 c$ for $\mathrm{C} 2$.

larly intriguing. This invokes the HST-1 region as a possible site of the $\mathrm{TeV}$ gamma-ray production as originally claimed based on the 2005 event. However, the short time scales of the variabilities of the $\mathrm{TeV}$ flares raise a severe question on this scenario [23]. Moreover, the TeV events in 2008 and 2010 seems to be correlated with the radio/Xray flux enhancements at the jet base [20, 21, 39, 42] (Figure 8). These facts tend to favor the jet base scenario more naturally, at least for the cases in 2008 and 2010.

Motivated by these situations, we decided to embark on a new monitoring project for M87 using VERA from October 2010. This project partly consists of the GENJI programme operated at $22 \mathrm{GHz}$ (Gamma-ray Emitting Notable AGN Monitoring with Japanese VLBI; for more details see [65]), but we are additionally conducting dedicated sessions for M87 (both at 22 and $43 \mathrm{GHz}$ with the dual-beam astrometry mode). Eventually, this monitor program achieves a very dense sampling interval (typically every two weeks but occationally as small as a few days to 1 week) throughout the year. The aim of this project is to reveal (1) the detailed radio lightcurve of the jet base, (2) the jet kinematics near the black hole, (3) the nuclear opacity and the magnetic field strength based on the core shift astrometry, and (4) the possible connections of these properties to the gamma-ray activities.
To describe the performance of this project, we show a preliminary result on the proper motion measurements for the jet base between September 2011 and June 2012 (Figure 9). During this period we observed M87 more than 20 epochs and for most of these data we consistently detected a few knotty features at $(0.5 \sim a$ few $)$ mas downstream of the radio core. Thanks to the dense sampling, we successfully track the proper motions of these features, demonstrating that the project has a nice capability to probe the detailed evolution at the M87 jet base. Our preliminary measurements indicates subluminal speeds around $(0.4 \sim 0.6) c$ for these features, which are significantly slower than the observed superluminal speeds in HST-1. These values are similar to those previously suggested by the year-scale-interval monitoring with VLBA $43 \mathrm{GHz}$ [28], but quite faster than the quasi-stationary motions measured by the month-scale-interval monitoring with VLBA $15 \mathrm{GHz}$ [30]. We have not confirmed yet any superluminal components in this region.

During the period of the VERA monitoring in 2012, M87 showed an elevated flux level at TeV gammaray [66]. Our preliminary analysis of the radio lightcurve found a curious flux increase from the core both at 22 and $43 \mathrm{GHz}$ that seems to be coincident with the gamma-ray 
event. These results will be presented in the forthcoming paper (Hada et al. in preparation).

\section{Summary and future prospects}

In this contribution we have reviewed some of our recent studies for M87 based on the multi-frequency and multiepoch VLBI observations. The astrometric measurements of the core shift indicate that the M87 core is nearly coincident with the central black hole, which is consistent with the previous counter jet detections. As a result, this confirms that the M87 jet is indeed launched with a wide opening angle. The launched jet is subsequently collimated into a parabolic shape over the distance of $\sim 10^{5} R_{\mathrm{s}}$, possibly with a transition of the collimation profile around $\sim 10 R_{\mathrm{S}}$ to $\sim 100 R_{\mathrm{S}}$ from the black hole, which could be related to the emergence of the fast-magnetosonic point. Regarding the jet kinematics, our intensive monitoring programs with VERA and EVN revealed a subluminal motions near the jet base, while the superluminal components in the HST-1 region with the possible exsistence of the stationary feature at the upstream edge of HST-1. Considering these structural/kinematical results together, one may deduce the following scenario as the nature of the M87 jet; the jet is initially launched magnetically, and subsequently accelerated and collimated down to the location of HST-1. At the end of the acceleration and collimation region, the M87 jet creates a recollimation shock that is observed as the stationary feature in HST-1. Eventually, this stationary region may result in a likely site of gamma-ray production, which can be associated with the blazar cores. However, the detailed ratio of the energy densities between magnetic field $\left(U_{\mathrm{B}}\right)$ and electrons $\left(U_{e}\right)$ at the jet base of M87 remains a major issue to be discussed [19]. To test the magnetically-driven scenario for the M87 jet, we are currently investigating the allowed range of $U_{e} / U_{\mathrm{B}}$ at the base of the M87 jet based on the fundamental furmula of SSA process (Kino et al. in preparation).

Our intensive monitoring programs with EVN and VERA are actively going on. These programs would further gain in imporntace by jointly performing with $\mathrm{TeV}$ telescopes, which will be able to constrain the TeV emission site more unambiguously. Finally, we also emphasize the importance of observations at mm-to-submm frequencies, because one can extract the emission even closer to the black hole thanks to the smaller opacity of synchrotron emission. We are the first group observing the M87 jet with ALMA, and are revealing the detailed mmsubmm spectral properties for the first time (Doi et al. in preparation). By collaborating with the highest resolution study with the Event-Horizon-Telescope [32], these data will yield a crucial insight into the physical properties for the jet launch region.

\section{Acknowledgements}

The author acknowledges all of the collaborators involved with the projects presented in this contribution. The VeryLong-Baseline-Array is operated by National Radio Astronomy Observatory, a facility of the National Science
Foundation, operated under cooperative agreement by Associated Universities, Inc. This work was partially supported by KAKENHI (24340042 and 24540240). This work made use of the Swinburne University of Technology software correlator [67], developed as part of the Australian Major National Research Facilities Programme and operated under license. We are grateful to the staff of all the VERA stations for their assistance in observations. This research has made use of data from the MOJAVE database that is maintained by the MOJAVE team [68]. Part of this work was done with the contribution of the Italian Ministry of Foreign Affairs and University and Research for the collaboration project between Italy and Japan.

\section{References}

[1] Meier, D. L., Koide, S., \& Uchida, Y., Science 291, 84 (2001)

[2] Koide, S., Shibata, K., Kudoh, T., \& Meier, D. L., Science 295, 1688 (2002)

[3] McKinney, J. C., \& Gammie, C. F., ApJ 611, 977 (2004)

[4] McKinney, J. C., MNRAS 368, 1561 (2006)

[5] Komissarov, S. S., Barkov, M. V., Vlahakis, N., \& Königl, A., MNRAS 380, 51 (2007)

[6] Tchekhovskoy, A., Narayan, R., \& McKinney, J. C., MNRAS 418, L79 (2011)

[7] Marscher A. P., Jorstad, S. G., D'Arcangelo, F. D., Smith, P. S., et al., Nature 452, 966 (2008)

[8] Spada, M., Ghisellini, G., Lazzati, D., \& Celotti, A., A\&A 325, 1559 (2001)

[9] Jordán, A., Côté, P., Blakeslee, J. P., et al., ApJ 634, 1002 (2005)

[10] Ford, H. C., Harms, R. J., Tsvetanov, Z. I., Hartig, G. F., Dressel, L. L. et al., ApJ 435, 27 (1994)

[11] Harms, R. J., Ford, H. C., Tsvetanov, Z. I., Hartig, G. F., Dressel, L. L., et al., ApJL 435, L35 (1994)

[12] Macchetto, F., Marconi, A., Axon, D. J., et al., ApJ 489, 579 (1997)

[13] Gebhardt, K., \& Thomas, J., ApJ 700, 1690 (2009)

[14] Gebhardt, K., Adams, J., Richstone, D. L., Tod R., et al., ApJ 729, 119 (2011)

[15] Walsh, J. L., Barth, A. J., Ho, L. C., \& Sarzi, M. ApJ 770, 11pp (2013)

[16] Owen, F. N., Hardee, P. E., \& Cornwell, T. J., ApJ 340, 698 (1989)

[17] Biretta, J. A., Sparks, W, B., \& Macchetto, F., ApJ 520, 621 (1999)

[18] Harris, D. E., Cheung, C. C., Biretta, J. A., Sparks, W. B., Junor, W. et al., ApJ 640, 211 (2006)

[19] Abdo, A. A., Ackermann, M., Ajello, M., Atwood, W. B., Axelsson, M. et al., ApJ 707, 55 (2009)

[20] Abramowski, A., Acero, F., Aharonian, F., Akhperjanian, A. G., Anton, G. et al., ApJ 746, 151 (2012)

[21] Acciari, V. A., Aliu, E., Arlen, T., Bautista, M., Beilicke, M. et al., Science 325, 444 (2009) 
[22] Aharonian, F., Akhperjanian, A., Beilicke, M., Bernlöhr, K., Börst, H.-G. et al., A\&A 403, L1 (2003)

[23] Aharonian, F., Akhperjanian, A. G., Bazer-Bachi, A. R., Beilicke, M. et al., Science 314, 1424 (2006)

[24] Aliu, E., Arlen, T., Aune, T., Beilicke, M., Benbow, W. et al., ApJ 746, 141 (2012)

[25] Junor, W., Biretta, J. A., \& Livio, M., Nature 401, 891 (1999)

[26] Dodson, R., Edwards, P. G., \& Hirabayashi, H., PASJ 58, 243 (2006)

[27] Ly, C., Walker, R. C., \& Wrobel, J. M., AJ 127, 119 (2004)

[28] Ly, C., Walker, R. C., \& Junor, W., ApJ 660, 200 (2007)

[29] Krichbaum, T. P., Graham, D. A., Bremer, M., et al., JPhCS 54, 328 (2006)

[30] Kovalev, Y. Y., Lister, M. L., Homan, H. H, \& Kellermann, K. I., ApJ 745, L28 (2007)

[31] Asada, N. \& Nakamura, M., ApJ 745, L28 (2012)

[32] Doeleman, S. S., et al., Science 338, 355 (2012)

[33] Reid, M. J., Biretta, J. A., Junor, W., Muxlow, T. W. B., \& Spencer, R. E., ApJ 336, 112 (1989)

[34] Cheung, C. C., Harris, D. E., \& Stawarz,Ł., ApJ 663, L65 (2007)

[35] Chang, C. S., Ros, E., Kovalev, Y. Y., \& Lister, M. L., A\&A 515, A38 (2010)

[36] Stawarz,Ł., Aharonian, F., Kataoka, J., Ostrowski, M.; Siemiginowska, A. et al., MNRAS 370, 981 (2006)

[37] Harris, D. E., et al. 2008, in ASP Conf. Ser. Vol.386, Extragalactic Jets: Theory and Observation from Radio to Gamma Ray (Rector T. A. \& De Young D. S., San Francisco, 2008), 80

[38] Harris, D. E., Cheung, C. C., Stawarz,Ł., Biretta, J. A., \& Perlman, E. S., ApJ 699, 305 (2009)

[39] Harris, D. E., Massaro, F., Cheung, C. C., Horns, D., Raue, M. et al., ApJ 743, 177 (2011)

[40] Hada, K., Doi, A., Kino, M., Nagai, H., Hagiwara, Y. et al., Nature 477, 185 (2011)

[41] Hada, K., Kino, M., Doi, A., Nagai, H., Honma, M. et al., ApJ 775, 70 (2013)

[42] Hada, K., Kino, M., Nagai, H., Doi, A., Hagiwara, Y. et al., ApJ 760, 52 (2012)

[43] Giroletti, M., Hada, K., Giovannini, G., Casadio, C., Beilicke, M. et al., A\&A 538, L10 (2012)

[44] Blandford, R. D., \& Königl, A., ApJ 232, 34 (1979)

[45] Marscher, A. P., in ASP Conf. Series Vol.386, Extragalactic Jets: Theory and Observation from Radio to Gamma Ray (Rector T. A. \& De Young D. S., San Francisco, 2008), 437

[46] Larionov, V. M., Jorstad, S. G., Marscher, A. P., Raiteri, C. M., Villata, M., A\&A 492, 389 (2008)

[47] Marscher, A. P., Jorstad, S. G., Larionov, V. M., Aller, M. F., Aller, H. D., et al., ApJ 710, 126 (2011)

[48] Agudo, I., Jorstad, S. G., Marscher, A. P., Larionov, V. M., et al., ApJ 726, 13 (2011)

[49] Königl, A., ApJ 243, 700 (1981)

[50] Lobanov, A. P., A\&A 330, 79 (1998)

[51] O'Sullivan, S. P., \& Gabuzda, D. C., MNRAS 400, 26 (2009)

[52] Sokolovsky, K. V., Kovalev, Y. Y., Pushkarev, A. B., \& Lobanov, A. P., A\&A 532, 38 (2011)

[53] Pushkarev, A. B., Hovatta, T., Kovalev, Y. Y., Lister, M. L., Lobanov, A. P. et al., A\&A 545, 113 (2012)

[54] Fromm, C. M., Ros, E., Perucho, M., Savolainen, T., Mimica, P. et al., A\&A 557, 105 (2013)

[55] Ghisellini, G., Tavecchio, F., \& Chiaberge, M., A\&A 432, 401 (2005)

[56] Perlman, E. S., Adams, S. C., Cara, M., Bourque, M., Harris, D. E. et al., ApJ 743, 119 (2011)

[57] Li, Z.-Y., Chiueh, T., \& Begelman, M. C., ApJ 394, 459 (1992)

[58] Vlahakis, D., \& Königl, A., ApJ 596, 1080 (2003)

[59] Tomimatsu, A., \& Takahashi, M., ApJ 592, 321 (2003)

[60] McKinney, J. C., Tchekhovskoy, A., \& Blandford, R., Science 339, 49 (2013)

[61] Broderick, A. E, \& Loeb, A., ApJ 697, 1164 (2009)

[62] Doeleman, S. S., Fish, V. L., Broderick, A. E., Loeb, A., \& Rogers, A. E. E., ApJ 695, 59 (2009)

[63] Marshall, H. L., Miller, B. P., Davis, D. S., Perlman, E. S., Wise, M. et al., ApJ 564, 683 (2002)

[64] Harris, D. E., Biretta, J. A., Junor, W., Perlman, E. S., Sparks, W. B. et al., ApJ 586, 41 (2003)

[65] Nagai, H., Kino, M., Niinuma, K., Akiyama, K., Hada, K. et al., PASJ 65, 24 (2013)

[66] Beilicke, M. et al., in ASP Conf. Series Vol.1505, High Energy Gamma-ray Astronomy: 5th International Meeting on High Energy Gamma-Ray Astronomy, 462 (2012)

[67] Deller, A. T., Brisken, W. F., Phillips, C. J., et al., PASP 123, 275 (2011)

[68] Lister, M. L., Cohen, M. H., Homan, D. C., Kadler, M., Kellermann, K. I. et al., AJ 138, 1874 (2009) 